\title{
Role of coronary artery calcium score for risk stratification in patients with non significant perfusion defects by myocardial perfusion single photon emission computed tomography
}

\author{
Márcio Vinícius Lins Barros ${ }^{1,2}$, Maria do Carmo Pereira Nunes ${ }^{3}$, \\ Gabriel Braga ${ }^{1}$, Daniel Rocha Rabelo ${ }^{1,4}$, Karen Magalhães ${ }^{2}$, \\ Fernanda Scaramello ${ }^{2}$, Maria Helena Albernaz Siqueira ${ }^{1}$ \\ ${ }^{1}$ Mater Dei Hospital, Belo Horizonte, MG, Brazil \\ ${ }^{2}$ School of Medicine, Faculdade de Saúde e Ecologia Humana, Vespasiano, MG, Brasil \\ ${ }^{3}$ School of Medicine, Universidade Federal de Minas Gerais, Belo Horizonte, MG, Brazil \\ ${ }^{4}$ School of Medicine, Faculdade Atenas, Paracatu, MG, Brazil
}

\begin{abstract}
Background: Myocardial perfusion scintigraphy (MPS) is an important diagnostic tool in the management of patients with suspected coronary artery disease (CAD). However, the presence of mild-moderate perfusion defects can be challenging and may lead to unnecessary cardiac catheterization. The coronary artery calcium (CAC) score is a method with excellent negative predictive value in the evaluation of $C A D$, but its role in this setting of patients has not been fully defined. This study aims to assess the potential of CAC in the prediction of cardiac adverse events in patients with suspected CAD with mild-moderate perfusion by MPS.
\end{abstract}

Methods and results: We conducted a cohort study in 292 patients presenting with mild-moderate perfusion defects by MPS undergoing a CAC measurement. The patients were followed for a mean of 34 months for occurrence of major cardiac adverse events (MACE). The majority of the patients (64.7\%) were male, mean age of $57.9 \pm 12.6$ years. During the follow-up there were 37 MACE. In multivariate Cox proportional hazards model, hypertension and CAC were independent predictors of MACE. The patients who presented a CAC score of $\geq 400$ had a high risk of MACE (HR 20.9; 95\% CI 4.79-91.42; $p<0.001$ ). Kaplan-Meier curve showed a significant difference (log-rank $\chi^{2} ; p<0.001$ ) using CAC scores in predicting MACE. Conclusions: CAC score carries a powerful prognostic value in predicting adverse events in patients with suspected CAD and MPS with mild-moderate perfusion defects and may be useful in risk stratification of these patients. (Cardiol J 2015; 22, 3: 330-335)

Key words: coronary artery disease, calcium score, myocardial perfusion SPECT

\section{Introduction}

Coronary artery disease (CAD) is an important cause of mortality and morbidity worldwide, with important socio-economic impacts [1]. The diagnosis of CAD takes into account the presence of symptoms, risk factors and complementary diagnostic methods that generally use stress

Address for correspondence: Dr Márcio Vinícius Lins Barros, Rua Carangola 57 apto 1201, Santo Antônio, 30330240 Belo Horizonte, MG, Brazil, e-mail: marciovlbarros@gmail.com

Received: 26.07.2014 Accepted: 21.09.2014 
mechanisms, with the goal of diagnosing the presence of myocardial ischemia. Among these, myocardial perfusion imaging (MPI) using single photon emission computed tomography (SPECT) plays an important role in the diagnosis and prognosis of CAD. However, the occurrence of positive results may be due to various clinical situations beyond obstructive coronary atherosclerosis [2]. SPECT studies with non-significant perfusion defects for myocardial ischemia may represent a challenge, sometimes leading to unnecessary indication for cardiac catheterization. A recent multicenter study demonstrated that, based on current algorithms used for the evaluation of patients with suspected $\mathrm{CAD}$, about $40 \%$ of patients undergoing coronary arteriography showed no coronary obstruction [3]. Coronary computed tomography angiography (CCTA) is an important method in the evaluation of the coronary arteries with high accuracy in the diagnosis and prognosis of coronary heart disease. Although coronary artery calcium (CAC) scoring has an established role in risk-stratifying asymptomatic patients at intermediate risk of CAD, its utility in the evaluation of this specifically group is uncertain [4-9]. The purpose of this study is to assess the potential of CAC in the prediction of cardiac adverse events in patients with suspected $\mathrm{CAD}$ with mild-moderate perfusion by MPI.

\section{Methods}

From January 2008 to July 2013, 804 consecutive patients with suspected $\mathrm{CAD}$, who underwent CCTA were prospectively enrolled.

Among them, 292 patients had SPECT with non-significant perfusion deficit (defined as a summed stress score [SSS] between 4 and 12) and were selected for this study. The exclusion criteria were: known CAD, acute coronary syndrome, cardiac arrhythmia, allergy to iodinated contrast material and renal failure.

Informed consent was obtained from all patients and the Ethics Committee of the institution approved the study.

Upon admission, each individual completed a collection of standardized data on the presence of cardiac risk factors. Systemic arterial hypertension was defined as a documented history of high blood pressure or treatment with antihypertensive medications. Diabetes mellitus was defined by a diagnosis of diabetes formerly carried out by a physician and/or use of insulin or oral insulinlowering medication. Dyslipidemia was defined as known dyslipidemia or current treatment with lipid-lowering medication. A positive history of smoking was defined as smoking or smoking cessation within 3 months of the examination. Family history of coronary heart disease was defined as the presence of $\mathrm{CAD}$ in first-degree relatives younger than 55 (men) or 65 (women) years of age.

\section{Cardiac CT imaging protocol}

All CT scans were performed on a 64-slice scanner with a $0.4 \mathrm{~s}$ rotation time (Aquilion multi-64-slice system, Toshiba Medical Systems). Non-enhanced CT scan for calcium scoring was performed from the level of tracheal bifurcation to the diaphragm using the following parameters: $120 \mathrm{KVp}, 300 \mathrm{~mA}, 0.25 \mathrm{~s}$, slice thickness of $3 \mathrm{~mm}$, and intervals of $3 \mathrm{~mm}$. The calcium scores of each area at each vessel were calculated at an offline commercially available workstation with dedicated software (Software Vitrea 2 V3.9.0.1, MN, USA) and the scores were quantified by the scoring algorithm proposed by Agatston et al. [10], and calcium scores were classified into the following categories: $0=0 ; 1=1-99 ; 2=100-399$ and $3 \geq 400$.

Patient adverse clinical outcomes were obtained through telephone interviews and were classified as the occurrence of: 1) cardiac death, 2) myocardial infarction or 3) need of revascularization.

\section{Statistical analysis}

The demographic and clinical characteristics of the study population were expressed as numbers and percentages for categorical variables and as mean and standard deviation for continuous variables. The variables were compared using $\chi^{2}$ test for categorical variables and Student's t-test for continuous variables. To satisfy the assumption that the events are independent, the recurrence of cardiac events in one participant was not included in the analysis.

Cox regression model was used to assess the value of clinical variables and $\mathrm{CAC}$ in predicting cardiac events. Initially, the univariable analysis of clinical characteristics and $\mathrm{CAC}$ measurement was performed to identify potential predictors. Hazard ratios (HR) were calculated with a $95 \%$ confidence interval (CI) as an estimate of risk associated with a particular variable. Subsequently, the multivariable analysis was performed including all variables selected in the univariate analysis. Cumulative event-free survival rates as a function over time were obtained by the Kaplan-Meier method. Cardiac event-free survival curves were compared using the log-rank test. Statistical analyses were performed using the SPSS software (version 18.0, SPSS Inc., Chicago, Illinois) and $\mathrm{p}<0.05$ was considered statistically significant. 


\section{Results}

At enrollment, 334 patients were initially selected, but 16 were excluded for indication of postcoronary artery bypass grafting (CABG) surgery and 26 for post-angioplasty evaluation. In total, 292 consecutive patients were included in the study; $64.7 \%$ were men, with a mean age of $57.9 \pm 12.6$ years. The general characteristics of the patients are summarized in Table 1 . The indications for MPI assessment were as follows: evaluation of chest pain in $95(32.5 \%)$ patients, positive stress test in $97(33.2 \%)$; asymptomatic patients with 2 or more risk factors in $79(27 \%)$ patients, and other causes in $21(7.2 \%)$ patients.

CAC score of 0 was presented in $45.5 \%$ of the patients, $1-100$ score in $26.7 \%, 101-400$ score in $14.4 \%$ and $13.4 \%$ had CAC score more than 400 . CAC 0 score had negative predictive value (NPV) of $97.5 \%$ for non-occurrence of events.

Table 1. Baseline characteristics of the study population.

\begin{tabular}{lc}
\hline Age [years] & $57.9 \pm 12.6$ \\
Male sex & $157(64 \%)$ \\
Smoking & $88(30.1 \%)$ \\
Hypertension & $169(57.9 \%)$ \\
Diabetes mellitus & $57(19.5 \%)$ \\
Dyslipidemia & $179(61.3 \%)$ \\
Familial history & $170(58.2 \%)$ \\
Coronary artery calcium score: & \\
$\quad 0$ & $133(45.5 \%)$ \\
$1-99$ & $78(26.7 \%)$ \\
$100-399$ & $39(13.4 \%)$ \\
$\geq 400$ & $42(14.4 \%)$ \\
\hline
\end{tabular}

Mean follow-up was 34 (range 3-66) months, carried out in $264(90.1 \%)$ patients. To determine whether loss at follow-up would influence the results, we performed a comparative analysis between the groups with and without follow-up, and no differences regarding age, sex, smoking status, hypertension, family history, dyslipidemia and diabetes were found.

A total of 37 events occurred during the follow-up and only those with adverse clinical outcomes occurring at least 3 months after followup were selected. Three patients died of cardiac cause, myocardial infarction occurred in 9 patients; 25 patients underwent coronary revascularization, with percutaneous coronary intervention being performed in 23 patients, while the remaining 2 patients underwent CABG. The decision for revascularization was based on worsening of angina and/or ischemia at noninvasive testing. Univariate analysis of clinical characteristics and at CCTA to predict adverse clinical outcomes is shown in Table 1. Variables that were significant in the univariate analysis were included in the multivariate analysis. Independent predictors of cardiac events obtained in the multivariate analysis are shown in Table 2.

In multivariate Cox proportional hazards model, CAC and hypertension were independent predictors of major cardiac adverse events (MACE; Table 3). A CAC score of $\geq 400$ was associated with a high risk of MACE (HR 20.9; 95\% CI 4.79-91.42; $\mathrm{p}<0.001$ ). Kaplan-Meier curve (Fig. 1) showed a significant difference (log-rank $\left.\chi^{2} ; \mathrm{p}<0.001\right)$ using CAC scores in predicting MACE. Event-free survival rate at 1,2 and 4-year follow-up was $99 \%$, $95 \%$ and $95 \%$ in patients with CAC 0-99 (groups 0 and 1 ), in comparison with $97 \%, 83 \%$, and $49 \%$ in those who had a CAC $\geq 400$ (group 3).

Table 2. Baseline characteristics of the study population according to the occurrence of cardiac events.

\begin{tabular}{lcccc}
\hline Baselines values & $\begin{array}{c}\text { Patients without } \\
\text { events }(\mathbf{n}=266)\end{array}$ & $\begin{array}{c}\text { Patients with } \\
\text { events (n = 37) }\end{array}$ & $\begin{array}{c}\text { Hazard ratio (95\% } \\
\text { confidence interval) }\end{array}$ & $\begin{array}{c}\text { P } \\
\text { Age [years] }\end{array}$ \\
\hline $56.8 \pm 12.3$ & $65.8 \pm 13.1$ & $1.013(1.009-1.064)$ & 0.008 \\
Male & $144(63 \%)$ & $26(70 \%)$ & $0.743(0.364-1.515)$ & 0.41 \\
Tabagism & $66(28 \%)$ & $13(36 \%)$ & $1.921(0.960-3.843)$ & 0.07 \\
Dyslipidemia & $135(60 \%)$ & $27(75 \%)$ & $1.885(0.881-4.031)$ & 0.10 \\
Hypertension & $120(53 \%)$ & $30(83 \%)$ & $4.476(1.962-11.481)$ & 0.000 \\
Sedentarism & $94(41 \%)$ & $13(36 \%)$ & $0.758(0.374-1.534)$ & 0.44 \\
Diabetes & $38(17 \%)$ & $12(33 \%)$ & $2.275(1.128-4.589)$ & 0.02 \\
Family history & $135(60 \%)$ & $16(44 \%)$ & $0.780(0.397-1.532)$ & 0.47 \\
Coronary artery calcium score 1 & $63(28 \%)$ & $7(19 \%)$ & $3.050(0.786-11.838)$ & 0.107 \\
Coronary artery calcium score 2 & $32(14 \%)$ & $9(24 \%)$ & $5.400(1.416-20.591)$ & 0.014 \\
Coronary artery calcium score 3 & $15(7 \%)$ & $18(49 \%)$ & $18.715(5.496-63.734)$ & 0.000 \\
\hline
\end{tabular}


Table 3. Multivariate logistic regression model for predicting cardiac events.

\begin{tabular}{lccc}
\hline Variables & Hazard ratio & 95\% confidence interval & P \\
\hline Hypertension & 3.027 & $1.212-7.560$ & 0.018 \\
Coronary artery calcium score 0/1 & 4.531 & $0.939-21.850$ & 0.06 \\
Coronary artery calcium score 0/2 & 5.879 & $1.220-28.338$ & 0.027 \\
Coronary artery calcium score 0/3 & 20.909 & $4.789-91.420$ & 0.000 \\
\hline
\end{tabular}

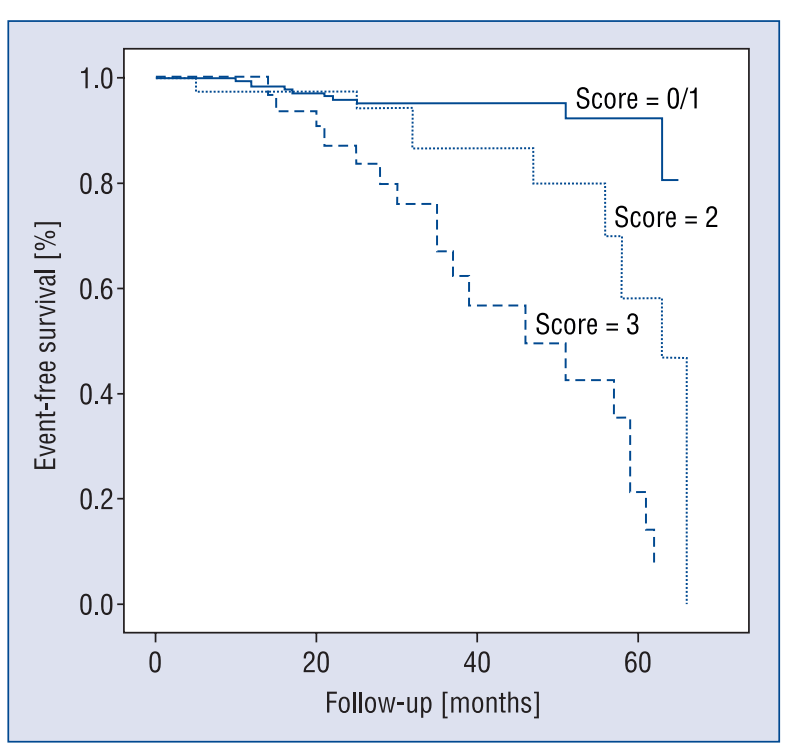

Figure 1. Kaplan-Meier survival curves comparing event-free survival rates according to the coronary artery calcium score groups $(0 / 1,2$ and 3$)$.

\section{Discussion}

In the present study, we found that CAC score poses an incremental value in the prognostic evaluation of patients with MPI studies presenting nonsignificant perfusion deficit and suspected CAD.

Since its introduction over 30 years ago, SPECT has become a mainstay for assessing diagnosis and prognosis of patients with suspected $\mathrm{CAD}$ [2]. For patients with normal results, the rate of cardiac death and myocardial infarction are less than $1 \%$. More extensive and severe perfusion defects are associated with up to $5 \%$ of the annual cardiac events rates [11-13]. The last subset is decidedly of high risk and favors the use of aggressive anti-ischemic therapy and consideration of coronary angiography and revascularization [14].

Patients with non significant perfusion defects $(\mathrm{SSS}<13)$ represent a group at intermediate risk for the occurrence of adverse events, with rates $1-3 \%$. Hachamovitch et al. [15] in a study involving 5,183 patients, followed by an average period of
642 days, showed that the annual rate of myocardial infarction was $0.5 \%$ in normal examinations, $2.7 \%$ had mild perfusion deficit, $2.9 \%$ for moderate deficit and $4.2 \%$ when there was severe impairment by SPECT. Doukky et al. [16], in a study encompassing 1,390 followed by an average period of 27 months demonstrated that patients with perfusion deficits in mild to moderate magnitude showed HR for adverse events of 2.3 (95\% CI 1.1-4.7, $\mathrm{p}=0.02$ ). On the other hand, non significant perfusion defects may be related to various clinical situations, such as the presence of endothelial dysfunction (an early marker of CAD), arrhythmias and myocardial hypertrophy without concomitant significant obstructive lesion, as well as a higher rate of equivocal studies due to attenuation artifacts, movement and gating, arrhythmias, conduction disturbances, errors of acquisition and post processing $[17,18]$. Given these considerations, the medical approach towards this group of patients can be challenging, requiring strategies to enable better stratification of these patients.

In our study, except hypertension, the conventional risk factors were not able to stratify these patients, which may be explained by their high prevalence, since the indication of SPECT is often associated to an intermediate pretest risk of CAD.

The presence of calcification in the coronary arteries is equivalent to the presence of atherosclerosis, which can be measured noninvasively using a CT [19]. The assessment of CAC is based on a non-contrast acquisition of a series of axial $\mathrm{CT}$ cuts with a $3 \mathrm{~mm}$ thickness covering the whole extension of the heart, with radiation doses from 0.9 to $1.1 \mathrm{mSv}$, defined as a hyper attenuating injury with signal intensity above $130 \mathrm{HU}$ and area of (at least $1 \mathrm{~mm}^{2}$ ). Several population studies have shown that CAC score correlates significantly with the occurrence of major cardiovascular events in the monitoring of medium and long term [20-23]. Its use as a diagnostic tool, however, is more controversial, and although it represents an excellent NPV in the exclusion of obstructive CAD (96-100\%), its positive predictive value (PPV) is moderate, which has been shown by more recent 
studies, particularly in younger patients or populations with a high prevalence of significant CAD. In addition to inappropriate PPV, CAC score also has insufficient NPV to exclude the presence of significant obstructive disease [19, 24].

Several studies have demonstrated the additive role of CCTA in patients undergoing SPECT. Abidov et al. [25] demonstrated the ability of re-stratification in patients with normal, abnormal or inconclusive SPECT regarding studies with normal coronary arteries, non-obstructive $\mathrm{CAD}$ and significant $\mathrm{CAD}$ (> 50\% stenosis) by CCTA. In this study, c.a. 53\% of patients with abnormal exams by scintigraphy showed normal coronary by CCTA. Cole et al. [26], in a study investigating 206 patients with SPECT showing non-significant perfusion abnormalities, as well as equivocal or inconclusive studies, have shown that only $32 \%$ of these patients underwent catheterization based on the results of the CCTA. However, this algorithm expresses a concern related to overexposure to radiation and a load of iodinated contrast, especially when patients are subjected to all 3 procedures (SPECT, CCTA and subsequent coronary angiography). Thus, the introduction of the CAC score, non-invasive method with low cost and radiation exposure and without contrast, may allow better diagnostic strategy in these patients. In our sample, the high NPV of the CAC score suggests that this method could be useful in prognostic stratification of these patients.

\section{Limitations of the study}

Among the limitations of this study, we mention the low rate of events, as well as a wide spectrum of different conditions associated with the examination indication. The exam results have the potential to influence the decision of revascularization indication and can change the outcome in this study. However, we considered an adverse clinical outcome only those that occurred after 3 months of follow-up (mean 34 [range 3-66] months). After this period, the indication for CABG was based on clinical decision, according to established guidelines. Nevertheless, the results of exams may be a confounding factor, being associated with the revascularization outcome. Studies of MPI were referred from different institutions, and this may be a cause of potential variability in the results. However, we believe that it may reflect the daily clinical practice more reliably in the real world. Furthermore, the study population was small, and studies performed on larger samples are clearly necessary to confirm these results.

\section{Conclusions}

In conclusion, CAC score carries a strong prognostic value in predicting adverse events in patients with suspected CAD and myocardial perfusion scintigraphy with mild-moderate perfusion defects and may be useful in risk stratification of these patients.

\section{Conflict of interest: None declared}

\section{References}

1. World Health Organization. Cardiovascular diseases. Fact sheet No. 317. Geneva 2007.

2. Marcassa C, Bax JJ, Bengel F et al. Clinical value, cost-effectiveness, and safety of myocardial perfusion scintigraphy: A position statement. Eur Heart J, 2008; 29: 557-563.

3. Patel MR, Peterson ED, Dai D et al. Low diagnostic yield of elective coronary angiography. N Engl J Med, 2010; 362: 886-895.

4. Schuijf JD, Pundziute G, Jukema JW et al. Diagnostic accuracy of 64-multislice computed tomography in the noninvasive evaluation of significant coronary artery disease. Am J Cardiol, 2006; 98: 145-148.

5. Hamon M, Giuseppe GB, Malagutti P et al. Diagnostic performance of multislice spiral computed tomography of coronary arteries as compared with conventional invasive coronary angiography: A meta-analysis. J Am Coll Cardiol, 2006;48: 1896-910.

6. Mowatt G, Cook JA, Hillis GS et al. 64-slice computed tomography angiography in the diagnosis and assessment of coronary artery disease: Systematic review and meta-analysis Heart, 2008; 94: 1386-1393.

7. Min JK, Shaw LJ, Devereux RB et al. Prognostic value of multidetector coronary computed tomographic angiography for prediction of all-cause mortality. J Am Coll Cardiol, 2007; 50: 1161-1170.

8. Chow BJW, Small G, Yam Y et al. The incremental prognostic value of cardiac CT in CAD using CONFIRM (CoroNary computed tomography angiography evaluation For clinical outcomes: An InteRnational Multicenter registry). Circ Cardiovasc Imag, 2011; 4: 463-472.

9. Barros MVL, Rabelo DR, Nunes MCP, Siqueira MHA. Coronary tomography for predicting adverse events in patients with suspected coronary disease. Arq Bras Cardiol, 2012; 99: 1142-1148.

10. Agatston AS, Janowitz WR, Hildner FJ, Zusmer NR, Viamonte M Jr, Detrano R. Quantification of coronary artery calcium using ultrafast computed tomography. J Am Coll Cardiol, 1990; 15: 827-832.

11. Bourque JM, Beller GA. Stress myocardial perfusion imaging for assessing prognosis: An update. J Am Coll Cardiol Cardiovasc Imag, 2011; 4: 1305-1319.

12. Thomas GS1, Miyamoto MI, Morello AP 3rd et al. Technetium $99 \mathrm{~m}$ sestamibi myocardial perfusion imaging predicts clinical outcome in the community outpatient setting. The Nuclear Utility in the Community (NUC) study. J Am Coll Cardiol, 2004; 21: 213-223.

13. Schinkel AFL, Boiten HJ, Sijde JN et al. 15-year outcome after normal exercise 99mTc-sestamibi myocardial perfusion imaging: What is the duration of low risk after a normal scan? J Nucl Cardiol, 2012; 19: 901-906. 
14. Shaw LJ, Narula J. Risk assessment and predictive value of coronary artery disease testing. J Nucl Med, 2009; 50: 1296-1306.

15. Hachamovitch R, Berman DS, Shaw LJ et al. Incremental prognostic value of myocardial perfusion single photon emission computed tomography for the prediction of cardiac death: Differential stratification for risk of cardiac death and myocardial infarction. Circulation, 1998; 97: 535-543.

16. Doukky R, Frogge N, Balakrishnan $G$ et al. The prognostic value of cardiac SPECT performed at the primary care physician's office. J Nucl Cardiol, 2013; 20: 519-528.

17. Abidov A, Raff GL. Value of coronary CTA in patients with known or suspected $\mathrm{CAD}$ and non-diagnostic initial myocardial perfusion testing: Current evidence and clinical considerations. J Nucl Cardiol, 2010; 17: 1101-1106.

18. Scanlon PJ, Faxon DP, Audet AM et al. ACC/AHA guidelines for coronary angiography: A report of the American College of Cardiology/American Heart Association Task Force on practice guidelines (Committee on Coronary Angiography): Developed in collaboration with the Society for Cardiac Angiography and Interventions. J Am Coll Cardiol, 1999; 33: 1756-1824.

19. Azevedo CF, Rochitte CE, Lima JAC. Coronary artery calcium score and coronary computed tomographic angiography for cardiovascular risk stratification. Arq Bras Cardiol, 2012; 98: 559-568.

20. Greenland P, Bonow RO, Brundage BH et al. ACCF/AHA 2007 clinical expert consensus document on coronary artery calcium scoring by computed tomography in global cardiovascular risk assessment and in evaluation of patients with chest pain: A report of the American College of Cardiology Foundation Clinical Expert Consensus Task Force (ACCF/AHA Writing Committee to Update the 2000 Expert Consensus Document on Electron Beam Computed Tomography). Circulation, 2007; 115: 402-426.

21. Budoff MJ, Achenbach S, Blumenthal RS et al. Assessment of coronary artery disease by cardiac computed tomography: A scientific statement from the American Heart Association Committee on Cardiovascular Imaging and Intervention, Council on Cardiovascular Radiology and Intervention, and Committee on Cardiac Imaging, Council on Clinical Cardiology. Circulation, 2006; 114: 1761-1791.

22. Greenland P, LaBree L, Azen SP, Doherty TM, Detrano RC. Coronary artery calcium score combined with Framingham score for risk prediction in asymptomatic individuals. JAMA, 2004; 291: 210-215.

23. Detrano R, Guerci AD, Carr JJ et al. Coronary calcium as a predictor of coronary events in four racial or ethnic groups. N Engl J Med, 2008; 358: 1336-1345.

24. Gottlieb I, Miller JM, Arbab-Zadeh A et al. The absence of coronary calcification does not exclude obstructive coronary artery disease or the need for revascularization in patients referred for conventional coronary angiography. J Am Coll Cardiol, 2010; 55: 627-634.

25. Abidov A, Gallagher MJ, Chinnaiyan KM, Mehta LS, Wegner JH, Raff GL. Clinical effectiveness of coronary computed tomographic angiography in the triage of patients to cardiac catheterization and revascularization after inconclusive stress testing: Results of a 2-year prospective trial. J Nucl Cardiol, 2009; 16: 701-713.

26. Cole JH, Chunn VM, Morrow JA, Buckley RS, Phillips GM. Cost implications of initial computed tomography as opposed to catheterization in patients with mildly abnormal or equivocal myocardial perfusion scans. J Cardiovasc Comput Tomography, 2007; 1: 21-26. 